\title{
Surface growth in laser-focused atomic deposition
}

\author{
E. Jurdik, Th. Rasing, and H. van Kempen \\ Research Institute for Materials, University of Nijmegen, Toernooiveld 1, 6525 ED Nijmegen, The Netherlands \\ C. C. Bradley and J. J. McClelland \\ Electron Physics Group, National Institute of Standards and Technology, Gaithersburg, Maryland 20899-8412
}

(Received 24 February 1999)

\begin{abstract}
We present calculations of surface growth in laser-focused nanostructure fabrication. We show that theoretical predictions of the structure profile's shape depend sensitively on the model used to describe the growth, and also on the parameters chosen within the model. This sensitivity illustrates that growth effects can play a major role in laser-focused atomic deposition, and also suggests that this process could be utilized for studies of surface growth mechanisms. [S0163-1829(99)06327-4]
\end{abstract}

The problem of surface growth in molecular-beam epitaxy (MBE) has attracted considerable research interest during the past two decades. ${ }^{1-4}$ The motivation for these studies originates from their importance for both fundamental research and technological applications. The latter requires growth of high-quality thin films or high contrast, high resolution, submicron structures that are used in nanoelectronic, optoelectronic and high-density magnetic storage devices. The key issue in the physics of MBE is the determination of what processes control the growth of material during and after deposition. Adsorption, diffusion, detachment and desorption all generally can play a role in a given situation. The task is to ascertain which of these dominate, as this governs the growth mode and hence the quality of an epitaxially grown material. The important growth processes can be studied experimentally using several techniques. ${ }^{2-4}$ For example, the motion of individual adatoms can be studied using a field ion microscope or a scanning tunneling microscope. Furthermore, surface growth kinetics can be measured with techniques such as low-energy electron diffraction or reflection high-energy electron diffraction.

In theoretical studies of surface growth, two different approaches have been developed. ${ }^{1}$ The continuous approach relies on a solution to a partial differential growth equation. It sees the surface on a coarse-grained scale larger than the typical interatomic distance. Atomistic growth models, on the other hand, rely on Monte Carlo calculations of atom-byatom deposition onto a fixed lattice of atom sites that are filled or left vacant according to a set of rules for bond formation and breaking. Separability of individual atomic-scale processes involved in the surface growth is the main advantage of this approach.

Laser-focused atomic deposition is a new technique for nanofabrication that can be used to grow periodic arrays of highly uniform nanometer-scale structures. ${ }^{5}$ Atoms from an atomic beam are deposited onto a substrate through a nearresonant laser standing wave. Such an arrangement of laser beams results in focusing of atoms to each of the nodes or antinodes of the laser intensity, depending on the sign of the detuning from the atomic resonance. The main advantages of this method are its MBE compatibility (i.e., nanostructure growth takes place in vacuo without any need for further physical or chemical processing) and its intrinsic massive parallelism, which results in a relatively large, periodically patterned surface area. These properties suggest that laserfocused atomic deposition could be a very attractive tool for surface growth and nanostructure studies. However, study of the roles played by surface growth and diffusion in laserfocused nanostructure formation remains a relatively unexplored area. ${ }^{6-8}$

To elucidate the underlying physical processes that govern the shape of laser-focused nanostructures, their evolution can be studied theoretically during and after the deposition. The same framework as in MBE growth can be applied; however, the unique property of a controlled, nonuniform atom flux distribution adds a new dimension to the problem. In typical theoretical studies of MBE, statistical quantities (e.g., surface roughness or island size distribution) are compared with corresponding measured quantities. ${ }^{1-4}$ For growth of laser-focused nanostructures, the shape of the nanostructure itself depends on the growth properties, and comparison can be made between theory and experiment on this more readily measurable quantity. Thus, studies can be carried out akin to earlier research in which corrugated surfaces were allowed to relax, ${ }^{9}$ with the added capability that the patterning and growth are combined into a single, ultra-high vacuum (UHV) compatible process.

In this report, we present calculations of the growth of a laser-focused nanostructure with the goal of examining the effects of growth and diffusion phenomena. Starting with the laser-focused atom flux calculated within a semiclassical trajectory tracing approach, ${ }^{10,11}$ we apply three different atomistic diffusion and growth models and examine the resulting profiles. We demonstrate that within the models applied, the nanostructure shape is strikingly sensitive to the kinetic parameters and the deposition time. Our results suggest that growth studies of laser-focused nanostructures under UHV conditions will show strong dependence on deposition rates and surface temperatures. While ideally our results would be compared with experimental results, all experimental studies to date have been carried out in high-vacuum conditions $\left(\sim 10^{-6} \mathrm{~Pa}\left[10^{-8} \mathrm{mbar}\right]\right),{ }^{5,7,8,12}$ and so measured profiles are likely to be influenced by contamination such as oxidation. Such comparisons will have to await future experiments conducted in UHV. 
The calculation of the initial incident flux used as a starting point in the present work is discussed in detail elsewhere ${ }^{8,11}$ Briefly, the motion of atoms in a laser standing wave is governed by a conservative optical potential ${ }^{13}$

$$
U(x, y, z)=\frac{\hbar \Gamma^{2}}{8 \Delta} \frac{I(x, y, z)}{I_{S}}
$$

provided the laser intensity $I(x, y, z)$ is relatively low and/or the detuning of the laser frequency from the atomic resonance $\Delta$ is relatively large, such that the population of the excited state is negligible. In Eq. (1), $I_{S}$ is the atomic saturation intensity, $\Gamma$ is the natural linewidth of the resonance transition and $\hbar$ is Planck's constant divided by $2 \pi$. Using this potential, the classical atomic equation of motion is integrated numerically for a large number of trajectories with varying initial conditions, giving a flux distribution at the substrate surface. Reasonable estimates of quantum (i.e., atomic diffraction) effects on the structure shape show that this semiclassical approach is a good approximation to the real situation. ${ }^{5}$ Furthermore, trends observed in experiments varying laser parameters indirectly demonstrate the validity of the trajectory tracing method. ${ }^{8}$

We consider the case of chromium atoms interacting with a laser tuned to the ${ }^{7} S_{3} \rightarrow{ }^{7} P_{4}$ transition $(\lambda=425.55 \mathrm{~nm})$, for which $\Gamma / 2 \pi=5 \mathrm{MHz}$, and $I_{S}=85 \mathrm{Wm}^{-2}$. The atoms are assumed to emerge from an effusion cell at $1900 \mathrm{~K}$ and to have a laser-collimated full-width at half maximum (FWHM) divergence of 0.16 mrad. $^{14}$ The detuning $\Delta / 2 \pi$ $=500 \mathrm{MHz}$ and the laser power $P=20 \mathrm{~mW}$ are adjusted in such a way that the minimum feature size is obtained on the substrate, located at the center of the Gaussian laser intensity profile, which has a $1 / e^{2}$ radius of $60 \mu \mathrm{m}$. The ground-state magnetic sublevel structure, the fraction of atoms relaxing to the ${ }^{5} D$ metastable state, as well as the presence of other chromium isotopes are all taken into account. Furthermore, the diffraction of the laser beam on the substrate is also considered. ${ }^{8}$ Trajectories of $10^{5}$ atoms are traced to obtain the atom flux distribution, and the result is shown in Fig. 1.

To fully characterize the flux shown in Fig. 1, one either needs its real-space profile as shown, or amplitudes of its spatial Fourier series. Nevertheless, it is useful to introduce two descriptive parameters, the width and the contrast. We define the former as the structure FWHM (above the background), while the latter is the ratio of the height (above the background) to the background. Both of these parameters depend on the assumed atom beam source and laser configuration. ${ }^{10,11}$ Since the profile in Fig. 1 represents the initial flux of atoms striking the surface, its width and contrast dictate the "ultimate" values achievable in a process with the given laser parameters and no surface diffusion or growth effects. In our case, Fig. 1 shows these "ultimate" values to be a FWHM of $13 \mathrm{~nm}$ and a contrast of 7 . It is interesting to note that though the values of the laser parameters can be controlled very well in an experiment, such narrow and high contrast nanolines have never been observed in chromium deposition studies. 5,7,8,12 This suggests that surface growth phenomena are playing a significant role in these studies.

The first surface growth model we have applied to laserfocused atomic deposition was introduced by Tamborenea

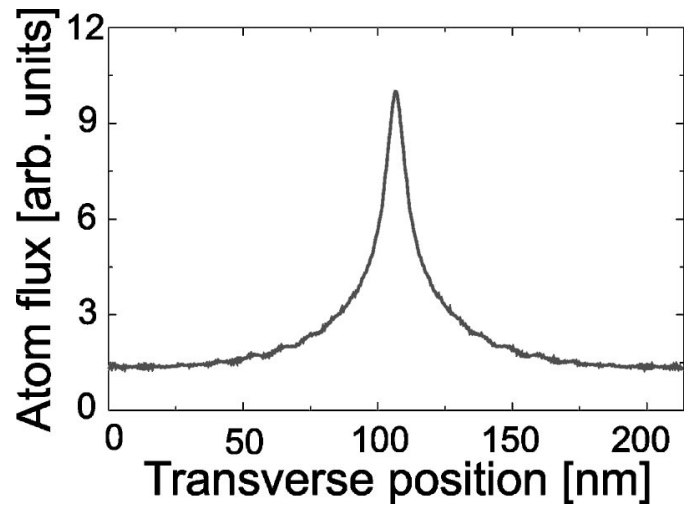

FIG. 1. Laser-focused chromium flux calculated within a semiclassical approximation by tracing trajectories of $10^{5}$ atoms exiting an oven at $1900 \mathrm{~K}$ and collimated to a FWHM divergence of 0.16 $\mathrm{mrad}$. The laser is detuned from the chromium two-level resonance by $+500 \mathrm{MHz}$. A laser power of $20 \mathrm{~mW}$ is used, as this gives the best focusing, given the Gaussian laser $1 / e^{2}$ radius of $60 \mu \mathrm{m}$.

and Das Sarma (TDS). ${ }^{15}$ In this model, the atoms are randomly deposited onto a one-dimensional substrate and stick only to the tops of already existing surface atoms. After each deposition event, the atoms having at maximum two nearest neighbors are allowed to break their bonds by a thermally activated process. After breaking its bonds, the atom hops to neighboring columns, provided that the initial site is as high as or higher than the final one. The diffusion $(n=1)$ and the step-edge detachment ( $n=2$ ) rates $R_{n=1,2}$ follow an Arrhenius behavior characterized by a system-dependent activation energy, and are given by ${ }^{15}$

$$
R_{n}=\frac{1}{R_{d}} \frac{k_{B} T_{S}}{\pi \hbar} \exp \left[-\frac{E_{0}+n E_{B}}{k_{B} T_{S}}\right],
$$

where $R_{d}$ is the deposition rate, $T_{S}$ is the surface temperature, $E_{0}$ is the "free atom" activation energy, $E_{B}$ is the bond breaking energy, $n$ is the number of nearest neighbors, and $k_{B}$ is Boltzmann's constant.

To examine the behavior of the TDS surface diffusion model we have performed deposition simulations for a wide range of values of $R_{1}$ and $R_{2}$, assuming an incident flux with an average deposition rate of 0.02 monolayers (ML) per second, distributed according to the profile shown in Fig. 1. The shape of the resulting profile is examined after total average coverages ranging from 5 monolayers (ML) to $150 \mathrm{ML}$. Periodic boundary conditions are used, as justified by the intrinsic periodicity of the laser focusing process.

After examining a number of cases, we are able to make the following qualitative observations. For $R_{1}=0$ and $R_{2}$ $=0$, simple random deposition applies. The shape of the nanostructure mirrors exactly the atom flux distribution for any amount of total coverage. If we set $R_{1}$ to a large number $\left(\gg 1\right.$ ) and $R_{2}$ equal to 0 , a flat terrace develops on top of the structure. The size of this terrace is dependent on the magnitude of $R_{1}$. However, considerable profile broadening is not observed in this regime. A significant increase in the structure FWHM and a decrease in the contrast with increasing average coverage takes place only for $R_{2} \gg 1$.

In Figs. 2(a) and 2(b) we show the structure evolution for a situation that might be typical for experiments with chro- 


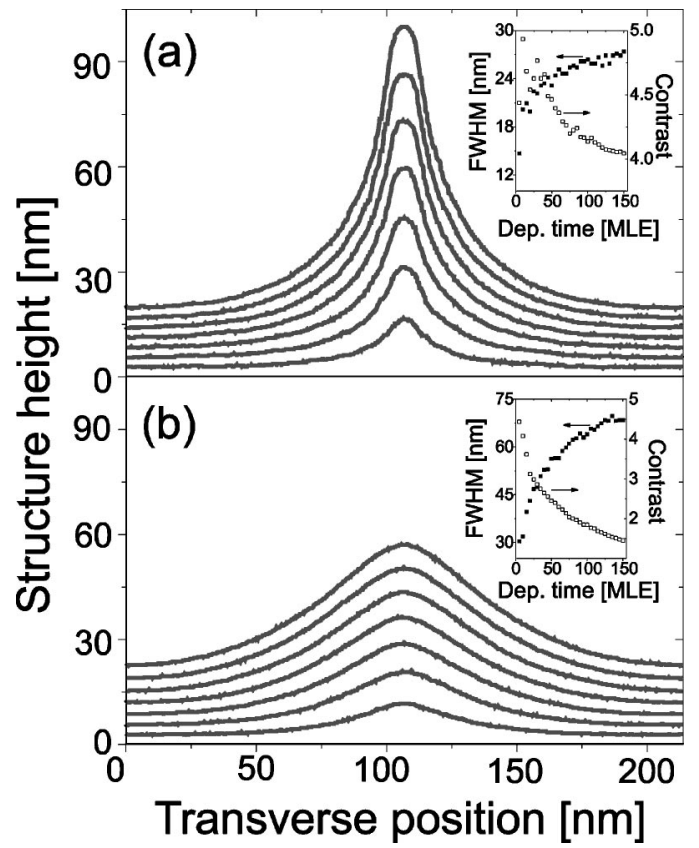

FIG. 2. Calculated TDS diffusion profiles for successive deposition times from 20 MLE to 140 MLE at 20 MLE intervals. (a) Substrate temperature $T_{S}=280 \mathrm{~K}$; (b) Substrate temperature $T_{S}$ $=300 \mathrm{~K}$. The evolution of the structure FWHM and contrast with deposition times from 5 MLE to 150 MLE at 5 MLE intervals is shown in the insets.

mium atoms. We set $E_{0}=0.55 \mathrm{eV}$ and $E_{B}=0.1 \mathrm{eV},{ }^{16}$ the lattice spacing to $0.249 \mathrm{~nm}$, and examine two different temperatures. Figure 2 (a) contains results for $T_{S}=280 \mathrm{~K}\left(R_{1}\right.$ $=1154, R_{2}=18$ ), and Fig. 2(b) shows the behavior for $T_{S}$ $=300 \mathrm{~K}\left(R_{1}=7456, R_{2}=156\right)$. In each figure, profiles are shown for total average coverage ranging from $20 \mathrm{ML}$ to 140 ML, at $20 \mathrm{ML}$ intervals. In the insets the evolution of the structure FWHM and contrast from $5 \mathrm{ML}$ to $150 \mathrm{ML}$, at 5 ML intervals, is presented. Comparing Figs. 2(a) and 2(b), we see that an increase of $T_{S}$ by just $20 \mathrm{~K}$ causes a very pronounced change in the structure's resolution and contrast. Recalling Eq. (2), we see that small changes in activation energies for diffusion and detachment would also result in a similarly strong effect.

This striking sensitivity to temperature and activation energy illustrates that within the framework of the TDS model, growth phenomena can have a dramatic effect on the shape of laser-focused nanostructures. Such sensitivity suggests not only that schemes for nanofabrication must take this into account, but also that laser-focused deposition studies can be used to reveal important information about surface diffusion kinetic parameters. Since there is no diffusion at low temperature $(<250 \mathrm{~K})$, measurements of the structure shape in this regime can be used to determine the incident flux. For higher temperatures, any measured change in the structure shape can be attributed to surface diffusion effects, allowing an in-depth investigation of these.

The second and third models implemented in our simulations are one-dimensional ballistic deposition models. ${ }^{1}$ In simple ballistic deposition (BD) the incident atom sticks to the first occupied site encountered, either directly below or laterally adjacent to it, and is subsequently not allowed to move. In ballistic deposition with relaxation (BDR), the

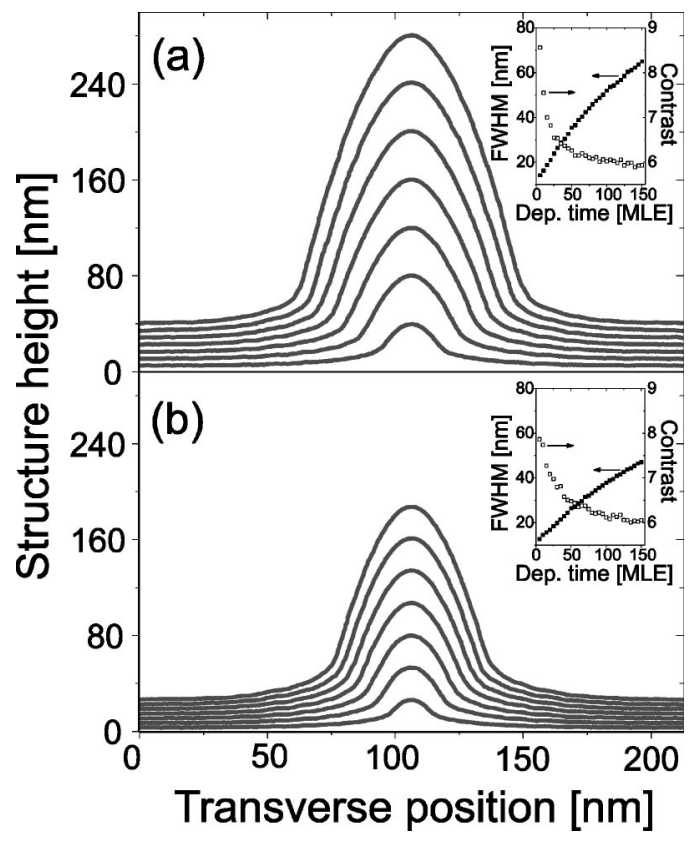

FIG. 3. Calculated nanostructure shapes for (a) BD and (b) BDR growth models. Shown are successive deposition times from 20 MLE to 140 MLE at 20 MLE intervals. The insets present the evolution of the structure FWHM and contrast with deposition times from 5 MLE to 150 MLE at 5 MLE intervals.

atom is allowed to relax to its nearest or next-nearest sites, the probability of movement being higher, the larger the coordination number of the new site. It should be noted that BD and BDR may not be realistic for describing MBE as they generally give rise to an unreasonably large number of voids and vacancies (especially for BD). ${ }^{1}$ Nonetheless, it is useful to study this type of growth because a certain number of these defects do occur in some systems, particularly for rough surfaces or for growth with high flux rates and/or a significant concentration of surface impurities.

The results of $\mathrm{BD}$ and $\mathrm{BDR}$ simulations are shown in Figs. 3(a) and 3(b). As in Fig. 2, the nanostructure evolution during the deposition is shown for deposition times of 20 ML equivalents (MLE) to 140 MLE at 20 MLE intervals. These data are a result of averaging along 100 independent statistical runs in order to obtain smooth curves. Comparing BD and BDR shapes with those of Fig. 2, we clearly see that the atomic growth mechanisms invoked in our simulations are reflected in the structure profile. Indeed, a significant difference is expected, because the two growth models belong to different universality classes. ${ }^{1}$ TDS is a linear surface diffusion model, ${ }^{15}$ while BD and BDR belong to the KardarParisi-Zhang (KPZ) universality class, ${ }^{17}$ with second order nonlinearity present in continuum versions of the model.

While the calculated structures for BD and BDR are similar, the average film thickness is different due to a different fractional volume of voids. In BDR atoms are allowed to move to their nearest or next-nearest sites to maximize the number of nearest neighbors. Allowing them to move more than once would result in a further collapse of voids and vacancies. The insets in both Fig. 3(a) and 3(b) show that the structure width increases as a function of the deposition time for both BD and BDR due to the strong lateral interaction between the atoms. This increase is faster for BD than for 
BDR. On the other hand, the contrast decreases with increasing deposition time, with both cases exhibiting the same trend. For low coverages $(<5 \mathrm{ML})$ the width measured for both of these models resembles the value of $13 \mathrm{~nm}$ from calculations of the atom flux. However, the low coverage contrast of about 8 is larger than the "ultimate" value of 7 derived from the atom-optically calculated flux. This increased contrast, which arises from a higher concentration of voids in the thicker part of the deposition, suggests that the real ultimate width and contrast might not only be dictated by the atom flux but also by surface growth effects. Such phenomena may play a crucial role in direct fabrication of nanostructures via atom optics.

In summary, we have applied three different atomistic growth models to laser-focused atomic deposition, starting with chromium flux calculated within a semiclassical trajectory tracing approach for experimentally realistic parameters.
We have shown that, within the models examined, the nanostructure profile depends strongly on the kinetic parameters and on the deposition time in our simulations. This suggests that laser-focused nanofabrication experiments done in UHV will be useful in discriminating between growth models. As this work progresses, a more thorough knowledge of the processes involved in laser-focused nanostructure fabrication will be gained, allowing growth of high resolution, high contrast, periodic nanostructures with a possible impact on a number of key technologies.

Useful discussions with M.D. Stiles, A.V. Petukhov, and W.L. Meerts are acknowledged. Part of this work was supported by the Stichting voor Fundamenteel Onderzoek der Materie (FOM), which is financially supported by the Nederlandse Organisatie voor Wetenschappelijk Onderzoek (NWO).
${ }^{1}$ A.-L. Barabási and H.E. Stanley, Fractal Concepts in Surface Growth, 1st ed. (Cambridge University Press, Cambridge, 1995).

${ }^{2}$ E.G. Seebauer and C.E. Allen, Prog. Surf. Sci. 49, 265 (1995).

${ }^{3}$ S.-L. Chang and P.A. Thiel, Crit. Rev. Surf. Chem. 3, 239 (1994).

${ }^{4}$ Mechanisms and Principles of Epitaxial Growth in Metallic Systems, edited by L.T. Wille, C.P. Burmester, K. Terakura, G. Comsa, and E.D. Williams, MRS Symposia Proceedings No. 528 (Materials Research Society, Pittsburgh, 1998).

${ }^{5}$ J.J. McClelland, R.E. Scholten, E.C. Palm, and R.J. Celotta, Science 262, 877 (1993).

${ }^{6}$ R.E. Behringer, V. Natarajan, and G. Timp, Appl. Surf. Sci. 104/ 105, 291 (1995).

${ }^{7}$ J.J. McClelland, R. Gupta, Z.J. Jabbour, and R.J. Celotta, Aust. J. Phys. 49, 555 (1996).

${ }^{8}$ W.R. Anderson, C.C. Bradley, J.J. McClelland, and R.J. Celotta, Phys. Rev. A 59, 2476 (1999).

${ }^{9}$ J.M. Blakely and H. Mykura, Acta Metall. 10, 565 (1962); P.S. Maiya and J.M. Blakely, J. Appl. Phys. 38, 698 (1967); K. Ya- mashita, H.P. Bonzel, and H. Ibach, Appl. Phys. 25, 231 (1981).

${ }^{10}$ K.K. Berggren, M. Prentiss, G.L. Timp, and R.E. Behringer, J. Opt. Soc. Am. B 11, 1166 (1994).

${ }^{11}$ J.J. McClelland, J. Opt. Soc. Am. B 12, 1761 (1995).

${ }^{12}$ U. Drodofsky, J. Stuhler, B. Brezger, Th. Schulze, M. Drewsen, T. Pfau, and J. Mlynek, Microelectron. Eng. 35, 285 (1997).

${ }^{13}$ J.P. Gordon and A. Ashkin, Phys. Rev. A 21, 1606 (1980).

${ }^{14}$ R.E. Scholten, R. Gupta, J.J. McClelland, R.J. Celotta, M.S. Levenson, and M.G. Vangel, Phys. Rev. A 55, 1331 (1997).

${ }^{15}$ P.I. Tamborenea and S. Das Sarma, Phys. Rev. E 48, 2575 (1993).

${ }^{16}$ The values chosen for $E_{0}$ and $E_{B}$ are essentially arbitrary, though they are not unreasonable for metal-on-metal homoepitaxy. The choice of the specific values used here was motivated by an attempt to model existing experiments. At temperatures near the experimental conditions these energies give a broadening of the profile that roughly matches the width seen in the experiments.

${ }^{17}$ M. Kardar, G. Parisi, and Y.-C. Zhang, Phys. Rev. Lett. 56, 889 (1985). 